\title{
Mejorando la docencia universitaria: introducción de metodologías activas de aprendizaje en aspectos fisiológicos de la conducta
}

\author{
Irene Cano-López - Universitat de València \\ Vanesa Hidalgo - Universitat de València \\ Esperanza González-Bono - Universitat de València
}

(iD) $0000-0001-9447-3244$

(iD) $0000-0001-8835-4505$

Recepción: 15.11.2019 | Aceptado: 20.11.2019

Correspondencia a través de ORCID: Irene Cano-López

0000-0001-9447-3244

Citar: Cano-López, I., Hidalgo, V. y González-Bono, E. (2019). Mejorando la docencia universitaria: introducción de metodologías activas de aprendizaje en aspectos fisiológicos de la conducta. REIDOCREA, 8, 411-421.

Financiación: Este estudio ha sido financiado por la Universitat de València (UV-INNOV17-18).

Resumen: Antecedentes. Los actuales planes de estudio permiten superar la tradicional división entre teoría y práctica en la docencia universitaria, artificial en términos formativos. Para que esta integración sea efectiva en la dinámica del aula, se requieren actividades que efectivamente integren conocimientos teóricos con habilidades y destrezas y que estimulen el trabajo autónomo del estudiante. Objetivos/Hipótesis. Este estudio se centra en analizar la satisfacción y percepción subjetiva de logro de competencias del alumnado universitario tras la implementación de metodologías activas de aprendizaje en una asignatura centrada en aspectos fisiológicos de la conducta. Método. Se implementaron metodologías activas de aprendizaje a 54 estudiantes (edad media: 24.17 años), incluyendo la realización de una fase experimental en el aula, la aplicación del método del caso, el desarrollo de estrategias de ludificación y la potenciación del alineamiento docente. Al final de la asignatura, se administró al alumnado un cuestionario de satisfacción. Resultados. La metodología docente fue unos de los aspectos mejor valorados por el estudiantado, seguido de los resultados del aprendizaje y, en menor medida, de la adquisición de competencias. Conclusiones. La implementación de metodologías docentes innovadoras en el ámbito universitario ha resultado satisfactoria para el estudiantado, en diferente medida en función del aspecto considerado.

Palabras clave: Innovación Educativa | Educación Universitaria

Improving higher education teaching: introduction of active learning methodologies in behavioral sciences

Abstract: Background. The current curricula allow to overcome the traditional division between theory and practice in higher education teaching, artificial in formative terms. For this integration to be effective in the classroom dynamic, activities are required that effectively integrate theoretical knowledge with skills and abilities and that stimulate the independent work of the student. Objectives/hypotheses. This study focuses on analysing the satisfaction and subjective perception of achievement of competencies of university students after the implementation of active learning methodologies in a subject focused on physiological aspects of behaviour. Method. Active learning methodologies were implemented for 54 students (mean age: 24.17 years), including an experiment in the classroom, the case method, the development of gamification strategies and the strengthening of constructive alignment. At the end of the course, a satisfaction questionnaire was administered to the students. Results. The learning methodology was one of the aspects most highly valued by the students, followed by learning outcomes and, to a lesser extent, the acquisition of competences. Conclusions. The implementation of innovative learning methodologies in the university environment has been satisfactory for the students, to a different extent depending on the aspect considered.

Keywords: Educational Innovation | Higher Education

\section{Introducción}

El Entorno Europeo de Educación Superior (EEES) recomienda la integración de tecnologías digitales y pedagógicas como un elemento integral en la educación superior (European Commission on New modes of learning and teaching in higher education, 2014). En este marco institucional, hemos experimentado grandes cambios a nivel social y psicopedagógico que han permitido el desarrollo de nuevas metodologías docentes 
que pretenden mejorar la docencia universitaria, enfatizando el protagonismo de los y las estudiantes en la construcción de su propio aprendizaje.

La sesión expositiva ha sido tradicionalmente la metodología más utilizada en la docencia universitaria. En esta línea, en muchas ocasiones, el aprendizaje universitario sigue considerándose como la recepción pasiva del conocimiento por parte de las y los estudiantes (Terenzini, 1999). De este modo, en muchas ocasiones, el aprendizaje está centrado en el personal docente y es dirigido por éste (Gil, 2001). Aunque esta metodología es útil para acercar la zona de desarrollo potencial del aprendizaje a la zona real de aprendizaje de los y las estudiantes, no es suficiente para que éstos desarrollen un aprendizaje significativo (Imbernon, 2009). De hecho, previos estudios han enfatizado que el alumnado aprende más cuando se implica en temas, problemas y actividades relacionadas con sus intereses y preocupaciones, se involucra en procesos de investigación, trabaja en contextos de colaboración, se enfrenta a situaciones de aprendizaje problemáticas, y reflexiona o evalúa su propio proceso de aprendizaje (Sancho y Hernández, 1999). Por tanto, la implementación de metodologías activas de aprendizaje que supongan una implicación más activa de los y las estudiantes en su proceso formativo es clave para favorecer su aprendizaje, así como para adaptarse al modelo educativo instaurado tras la reforma universitaria (Álvarez, González, Alonso, y Arias, 2014; Olmedo, 2013).

Este estudio pretende enfatizar las relaciones multidireccionales entre los y las estudiantes para que éstos sean los protagonistas de su propio aprendizaje. En este sentido, pretendemos aumentar la implicación del profesorado en el desarrollo de la asignatura, a fin de que el alumnado desarrolle un aprendizaje significativo de ésta, incluyendo como parte de esta, metodologías activas de aprendizaje que implican la creación de situaciones experimentales en el aula y la resolución de casos clínicos reales a los que un psicólogo podría enfrentarse en su vida profesional.

En este trabajo contemplamos la realización de diferentes actividades de aula dirigidas a favorecer la integración entre los conocimientos teóricos y la práctica, proponiendo una actividad investigadora, mediante la creación de situaciones experimentales en el aula, que permite extrapolar los resultados a la vida cotidiana y los hábitos saludables de los y las estudiantes. Con ello, se pretende que el estudiantado adquiera competencias como ser capaz de describir y evaluar procesos cognitivos, emocionales, psicobiológicos y conductuales, así como conocer y ajustarse a las obligaciones deontológicas de la Psicología, y profundizar en los fundamentos biológicos de la conducta humana y de las funciones psicológicas relacionadas con los procesos cognitivos, considerando la perspectiva de género, transversal a las competencias de la Titulación. Asimismo, pretende que el estudiantado ponga en práctica estrategias de investigación aplicada a los problemas de la profesión.

El cariz teórico-práctico de la dedicación presencial permite la implementación de las actividades de aula propuesta en esta experiencia de innovación docente, facilitando la integración entre conocimientos, competencias y destrezas. Por su parte, las estrategias metodológicas propuestas permiten la aplicabilidad directa de los contenidos, independientemente de su cariz teórico o práctico, a fin de facilitar la conexión de estas competencias con la realidad profesional y social. Para ello, se abordarán estrategias de participación activa que impliquen el saber hacer y estrategias de alineamiento docente para propiciar la coherencia entre objetivos docentes y evaluación. A su vez, se incluirán estrategias de ludificación como un mecanismo para incitar a la acción, promover el aprendizaje y resolver problemas (Kapp, 2012; Zichermann y Cunningham, 2011). Estudios previos han mostrado que estas estrategias pueden catalizar el cambio social, ya que tienen un carácter interactivo y transversal e impulsan comportamientos 
deseados para el desarrollo moral de las personas en pro del compromiso activo en la comunidad (Romero-Rodríguez, Torres-Toukoumidis, y Aguaded, 2017).

\section{Objetivos o hipótesis}

Este estudio se centra en analizar la satisfacción y percepción subjetiva de logro de competencias del alumnado universitario tras la implementación de metodologías activas de aprendizaje en una asignatura centrada en aspectos fisiológicos de la conducta.

\section{Métodos}

\section{Participantes}

En este estudio participaron 54 estudiantes universitarios españoles del último curso del Grado en Psicología (edad media: 24.17 años; rango: 21-51 años), de los cuales 42 eran mujeres $(77.8 \%)$ y 12 eran hombres $(22.2 \%)$. Los criterios de inclusión fueron los siguientes: a) estar matriculado en la asignatura "Psicoendocrinología" de Grado en Psicología en el curso 2017-2018; y b) firmar un consentimiento informado.

Respecto a las características de los participantes, la forma de inicio de la titulación fue a través de una prueba de acceso universitaria en el $72.2 \%$ de los casos ( $n=39$ ), un ciclo formativo superior en el $18.5 \%$ de los casos $(n=10)$, un programa internacional en el $5.6 \%(n=3)$, forma de acceso para titulados universitarios en un $1.9 \%(n=1)$, y forma de acceso para mayores de 25 años en un $1.9 \%(n=1)$. El $66.7 \%$ de los/as estudiantes no poseía ningún título de enseñanza superior $(n=36)$, el $24.07 \%$ contaba con estudios superiores no universitarios $(n=13)$, el $5.6 \%$ había cursado tres años de carrera universitaria $(n=3)$, el $1.9 \%$ poseía un título de estudios universitarios de ciclo largo $(n$ $=1)$, y el $1.9 \%$ contaba con un título de estudios universitarios de ciclo corto $(n=1)$. El $77.8 \%$ de los y las estudiantes no realizaba ningún trabajo remunerado $(n=42)$, el $11.1 \%$ trabajaba como media menos de 15 horas a la semana $(n=6)$, el $5.6 \%$ trabajaba como media 15 o más horas a la semana, y el 3\% no especificó esta información. Los estudios del padre fueron licenciado, ingeniero, arquitecto, estudios superiores militares o similares en un $22.2 \%$ de los/as estudiantes $(n=12)$, diplomado universitario o similar en un $7.4 \%$ del estudiantado $(n=4)$, bachillerato elemental o similar en un $18.5 \%(n=$ $10)$, bachillerato superior o similar en un $18.5 \%(n=10)$, primarios completos en un $14.8 \%(n=8)$, sin estudios en un $13.0 \%(n=7)$, y no especificados en un $5.6 \%(n=3)$. Los estudios de la madre fueron licenciado, ingeniero, arquitecto, estudios superiores militares o similares en un $18.5 \%$ de los y las estudiantes $(n=10)$, diplomado universitario o similar en un $18.5 \%(n=10)$, bachillerato elemental o similar en un $16.7 \%$ $(n=9)$, bachillerato superior o similar en un $16.7 \%(n=9)$, primarios completos en un $14.8 \%(n=8)$, sin estudios en un $9.3 \%(n=5)$, y no especificados en un $5.6 \%(n=3)$.

\section{Instrumentos}

Para evaluar la acción formativa se administró un cuestionario dirigido a la valoración por parte del alumnado de la satisfacción con el desarrollo de la asignatura y con las metodologías activas de aprendizaje utilizadas. Su administración aseguró el anonimato ya que no constaba ningún elemento identificativo, se realizó con el personal docente fuera del aula y fue recogido por un estudiante voluntario. Resumidamente, este cuestionario se compuso de 12 ítems relativos a la contribución que la asignatura había tenido para alcanzar las competencias (las 6 competencias específicas de la asignatura) y los resultados del aprendizaje (los 3 resultados del aprendizaje de la asignatura), así como el nivel de satisfacción sobre las metodologías docentes y su contribución al 
aprendizaje (3 ítems). Todos estos ítems fueron valorados mediante una escala visual de 10 puntos en la que, a menor puntuación, menor grado de contribución. Además, se incluyeron 5 cuestiones sobre el volumen de trabajo dedicado a la asignatura, tanto presencial como autónomamente. Respecto al trabajo presencial, se preguntó si se consideraba que el número de créditos de la asignatura respondía a la realidad y, en caso negativo, cuántos créditos se le otorgaría. Respecto al trabajo autónomo, se preguntó cuántas horas se habían invertido semanalmente en la asignatura y, de ellas, cuántas se dedicaron a trabajos y cuántas a estudiar contenidos. Por último, se realizaron 4 preguntas abiertas sobre posibles solapamientos de contenidos y los aspectos que consideraban mejor y peor en la asignatura, aunque por el contenido cualitativo de las respuestas, estas preguntas no se incluyen en el análisis.

Las calificaciones obtenidas por los/as estudiantes se consideraron tanto a nivel cuantitativo como cualitativo.

\section{Procedimiento}

Las acciones metodológicas que se describen a continuación se enmarcan en las siguientes líneas de actuación estratégica: el uso de entornos virtuales de aprendizaje, el fomento de participación de los estudiantes en la mejora de los procesos de enseñanza-aprendizaje, la realización de actividades que faciliten la conexión de los titulados y las tituladas con el mercado de trabajo, y la valoración y la transferencia del conocimiento y la investigación en materia de innovación educativa.

En este estudio se pusieron en marcha cuatro acciones metodológicas innovadoras en Ciencias de la Salud en un grupo de 54 estudiantes universitarios españoles del último curso de Psicología. Al final de la asignatura, pero antes de la evaluación final, se administró un cuestionario de satisfacción y de percepción subjetiva del logro de competencias, de manera voluntaria y anónima. Asimismo, como indicador objetivo, se compararon las calificaciones del grupo con implantación de estrategias innovadoras con un grupo de características similares en el que no se han puesto en práctica. A continuación, se describen las acciones metodológicas puestas en marcha y un detalle de la evaluación de la experiencia innovadora.

Aprendiendo a investigar: realización de un experimento en el aula. El objetivo de la presente actividad fue favorecer la integración entre los conocimientos teóricos y la práctica investigadora. Esta actividad consistió en la realización de un experimento en el aula, con una propuesta inicial de un diseño experimental y la organización de los y las estudiantes en equipos con responsabilidad y roles específicos para cada miembro del equipo. Cada equipo contó con un investigador principal, al que se le instruyó previamente, mediante tutorías, en los pormenores del diseño experimental, instrumentos de medida, e interpretación de resultados. Estos y estas estudiantes, a su vez, organizaron a los miembros de sus equipos en diferentes roles como: sujetos experimentales, investigadores 0 investigadoras responsables del registro e investigadores o investigadoras responsables de la manipulación experimental. De esta manera, el día de la práctica las funciones de cada estudiante estuvieron perfectamente delimitadas, permitiendo manejarse con grandes grupos, de aproximadamente 50 estudiantes.

Aprender haciendo: utilización del método del caso en el aprendizaje de trastornos psicoendocrinológicos. El objetivo de la presente práctica fue que el alumnado conociera las características psicofisiológicas asociadas a un caso real de depresión post-parto. Los objetivos específicos de la actividad fueron: a) conocer los síntomas de la depresión post-parto, b) analizar las alteraciones endocrinas en las hormonas sexuales y 
glucocorticoides que se producen en este trastorno, y c) diseñar un plan de tratamiento. El procedimiento para la realización de la práctica fue el siguiente: se establecieron grupos heterogéneos de entre cuatro y seis personas, dado que la literatura previa sugiere que el trabajo en un grupo heterogéneo permite contrastar las ideas de cada miembro del grupo, favorece la convivencia con personas distintas, y ayuda a mantener una relación productiva con los miembros del grupo (Reynolds, 1990). La actividad se estructuró de la siguiente manera. Se realizó una primera sesión expositiva para explicar conceptos básicos: cambios hormonales que se producen a lo largo del ciclo vital, y perfiles hormonales en el embarazo y la lactancia. En una segunda sesión, el personal docente formó los grupos de trabajo y se presentó el caso clínico a los y las estudiantes para que resolvieran el primer y el segundo objetivo (objetivos "a" y "b"). En una tercera sesión se explicó cómo realizar una búsqueda bibliográfica y se pidió al alumnado que resolviera el objetivo "c". Finalmente, se llevó a cabo la puesta en común con el gran grupo.

El alineamiento docente como estrategia para fomentar el interés de los y las estudiantes. A fin de alinear los tres elementos básicos del programa docente (objetivos, tareas y sistema de evaluación), las actividades anteriores se evaluaron mediante la entrega de un informe de grupo, la resolución de preguntas ante el gran grupo y la inclusión de dos preguntas cortas en el examen final de la asignatura (una sobre la interpretación de los resultados de un experimento similar al planteado en clase y otra sobre un caso clínico de características similares al caso planteado en case). Con ello, se pretendía que los y las estudiantes aplicaran las competencias adquiridas durante la asignatura a la interpretación de resultados de investigación en la materia que nos ocupa y a la resolución de casos clínicos reales.

La ludificación como estrategia para fomentar la motivación y la consolidación de conocimientos. Al finalizar cada uno de los temas, las alumnas y los alumnos respondieron unas cuestiones relacionadas con el tema mediante la web kahoot.it (https://kahoot.it/). De este modo, se pretendía favorecer su motivación y facilitar la consolidación de los conocimientos adquiridos a lo largo del tema. Al finalizar el curso, se realizó un ranking final basado en los resultados obtenidos en la totalidad de temas de la asignatura en este juego. Con ello, se pretendía reforzar positivamente el aprendizaje continuo del alumnado en un contexto de "juego", premiando su motivación sin poner en práctica castigos en los casos en los que no se adquirieran las competencias de alguno de los temas de la asignatura y, de este modo, evitando la frustración de los y las estudiantes.

\section{Análisis de datos}

Estudio descriptivo.

\section{Resultados}

El cuestionario fue respondido por un total de 44 alumnos y alumnas, sin diferenciar por sexo, del total de 54 estudiantes matriculados/as en el grupo. Sólo hubo dos datos faltantes, una persona no respondió al número de créditos que consideraba que debía tener la asignatura y otra persona no contestó al tercer ítem de metodologías docentes. Se calculó la media de puntuación para cada ítem.

El análisis de los resultados de esta encuesta muestra que, en lo relativo al nivel de contribución de la asignatura para alcanzar las competencias (Figura 1), las puntuaciones oscilaron entre 6.22 y 7.80 . Estas puntuaciones pueden interpretarse como aceptables, aunque mejorables. Sin embargo, cuando observamos las 
puntuaciones medias relativas a la contribución de la asignatura a lograr los resultados del aprendizaje, éstas oscilan entre 7.52 y 8.82 , que pueden considerarse sensiblemente más satisfactorios. Un detalle de las puntuaciones para cada ítem, tanto en lo que atañe a las competencias como a los resultados del aprendizaje, puede apreciarse en la Figura 2.

\section{COMPETENCIAS DE APRENDIZAJE}

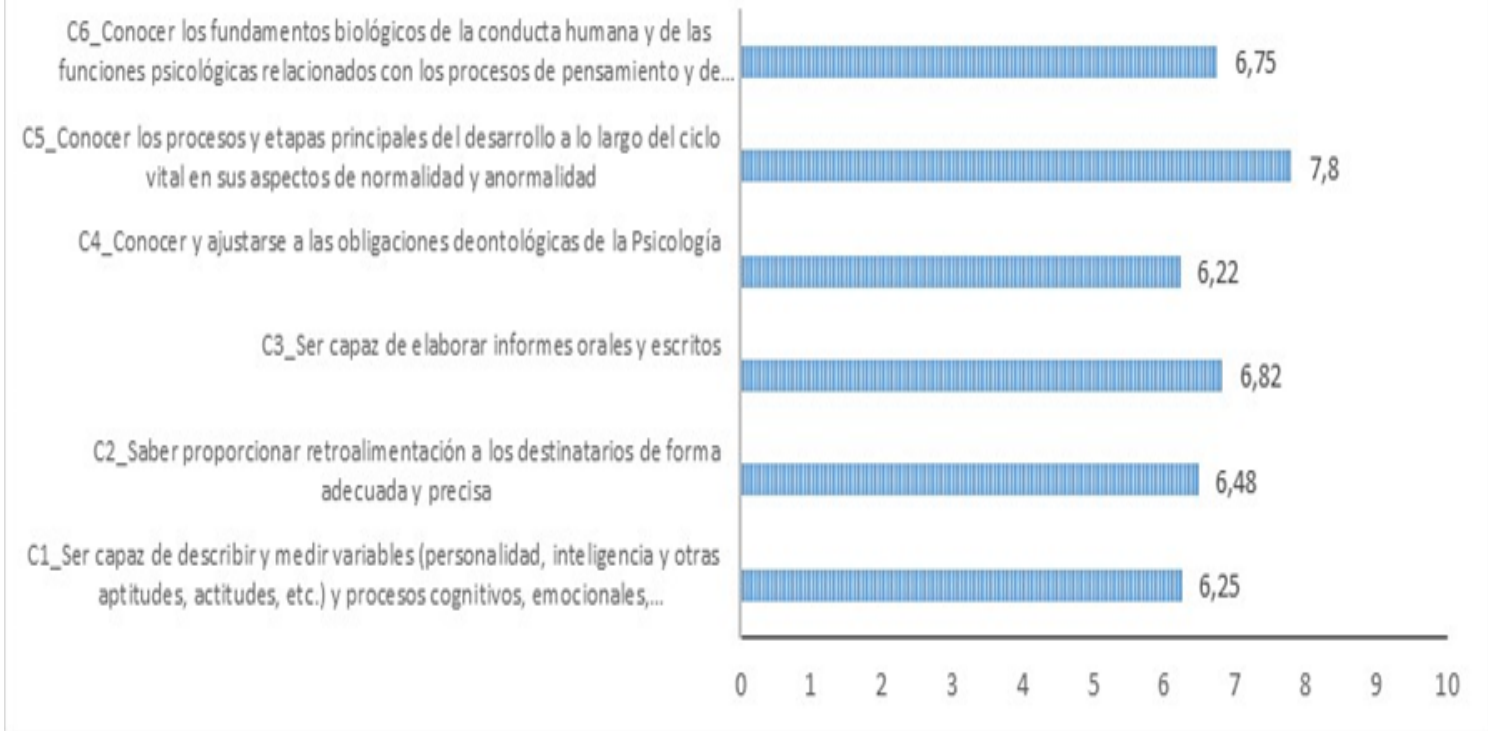

Figura 1. Puntuaciones en los ítems de contribución de la asignatura a cada una de las competencias.

\section{RESULTADOS DEL APRENDIZAJE}

R3_Discriminar entre alteraciones psicológicas primarias y secundarias a alter aciones endocrinas para establecer un diagnóstico diferencial

R2_Identificar y describir los patrones hormonales asociados al dimorfismo sexual, el estrés, el estado de ánimo, la conducta social y la función cognitiva.

R1_Describir los procesos hormonales a lo largo del ciclo vital

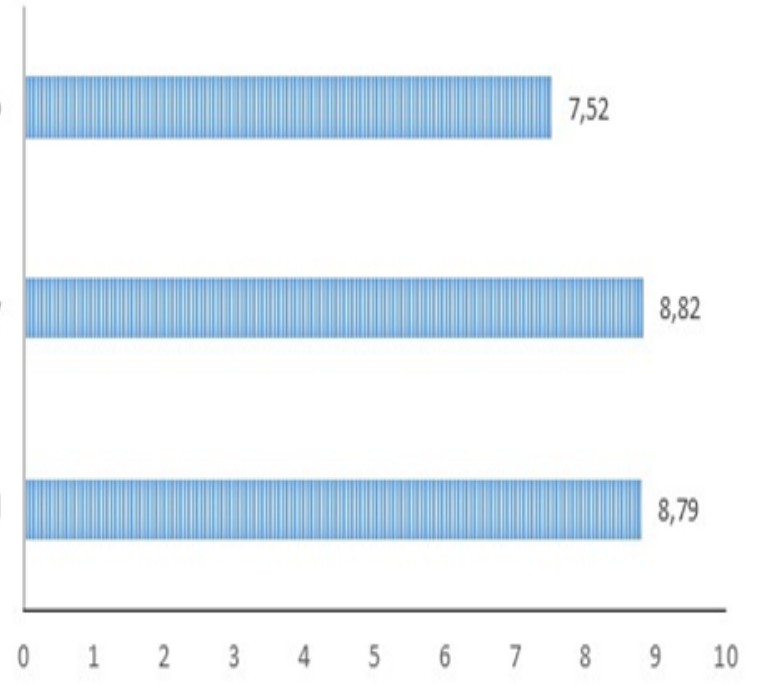

Figura 2. Puntuaciones en los ítems de contribución de la asignatura a cada uno de los resultados del aprendizaje contemplados. 
Respecto a las metodologías docentes, las puntuaciones oscilan entre 8.41 y 8.91 , lo que puede considerarse muy satisfactorio. Es posible que la inclusión, impulsada por la concesión del Proyecto de Innovación Educativa durante este curso, de nuevas metodologías docentes en esta asignatura haya favorecido una mayor satisfacción sobre este aspecto durante el proceso de enseñanza-aprendizaje. Un detalle de las puntuaciones de cada uno de los ítems puede apreciarse en la Figura 3.

\section{METODOLOGÍAS DOCENTES}

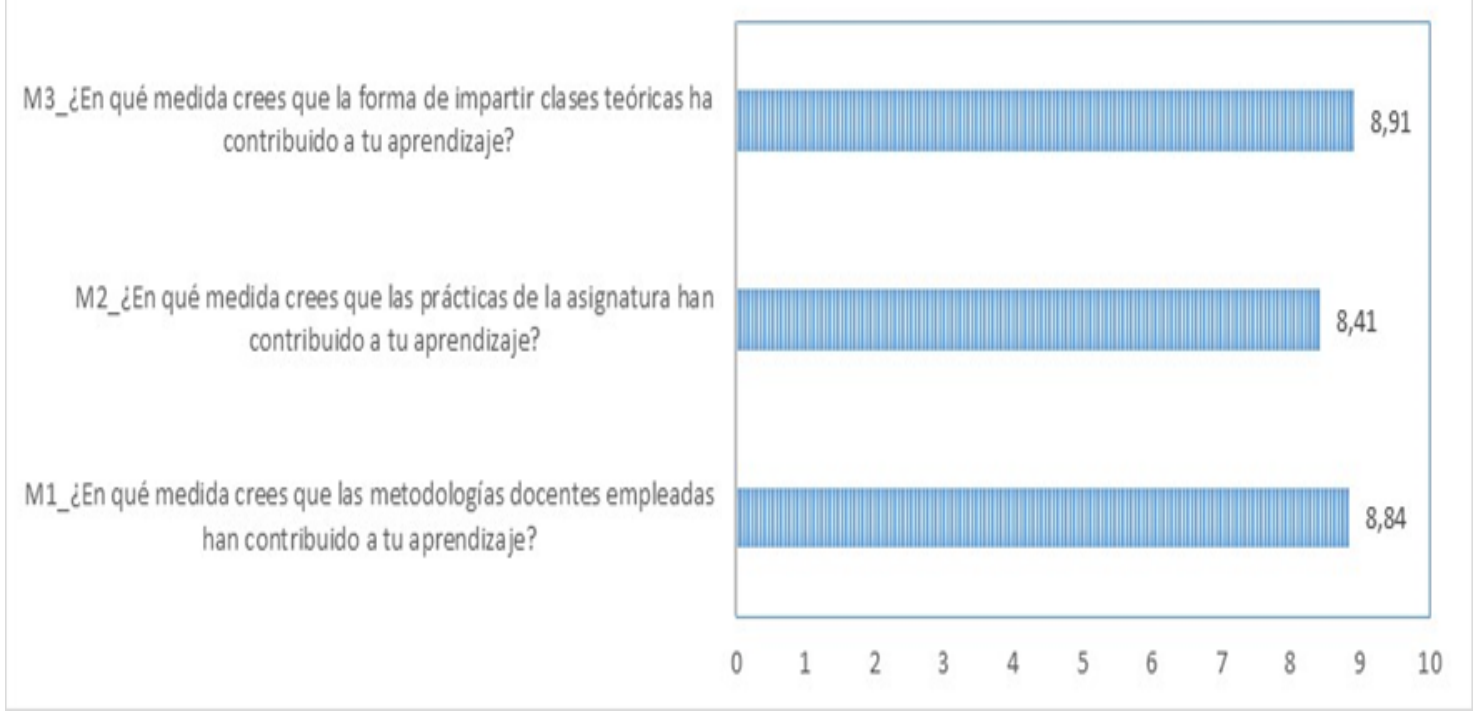

Figura 3. Puntuaciones en los ítems de contribución de las metodologías docentes al aprendizaje.

Respecto al volumen de trabajo, de acuerdo con la Guía Académica, hay una presencialidad de 45 horas mientras que se estima una dedicación de 67.5 horas de trabajo autónomo.

La presencialidad real en el aula se corresponde, como no podría ser de otra manera, con la indicada en la Guía Académica. Sin embargo, sólo nueve estudiantes (un 20.9\%) piensan que los créditos se ajustan a los contenidos. Por el contrario, 34 de los 43 estudiantes que han respondido a esta pregunta (un $79.1 \%$ ) piensan que no se ajusta. De ellos, un estudiante piensa que requiere 9 ECTS, otro cree que 10 ECTS, otro considera que 12 ECTS y los 31 restantes piensan que la asignatura requiere 6 ECTS. Considerando que siempre se termina el temario completo con las sesiones correspondientes a 4.5 ECTS, esta percepción puede implicar, entre otras posibilidades, que el alumnado considere que las clases son muy densas o que algunos contenidos se imparten de manera más superficial.

Sin embargo, esta percepción no se corresponde con el volumen de trabajo autónomo invertido por el alumnado (Figura 4). De hecho, la Guía Académica estima que el alumnado dedicará 67.5 horas autónomamente, mientras que las horas que el alumnado dedica a la asignatura fuera de clase sólo es de 49.2 horas, lo que supone una discrepancia media de 18.3 horas. Respecto a qué se dedica este tiempo, existe una proporción equilibrada entre el tiempo dedicado a trabajos y el dedicado al estudio. 


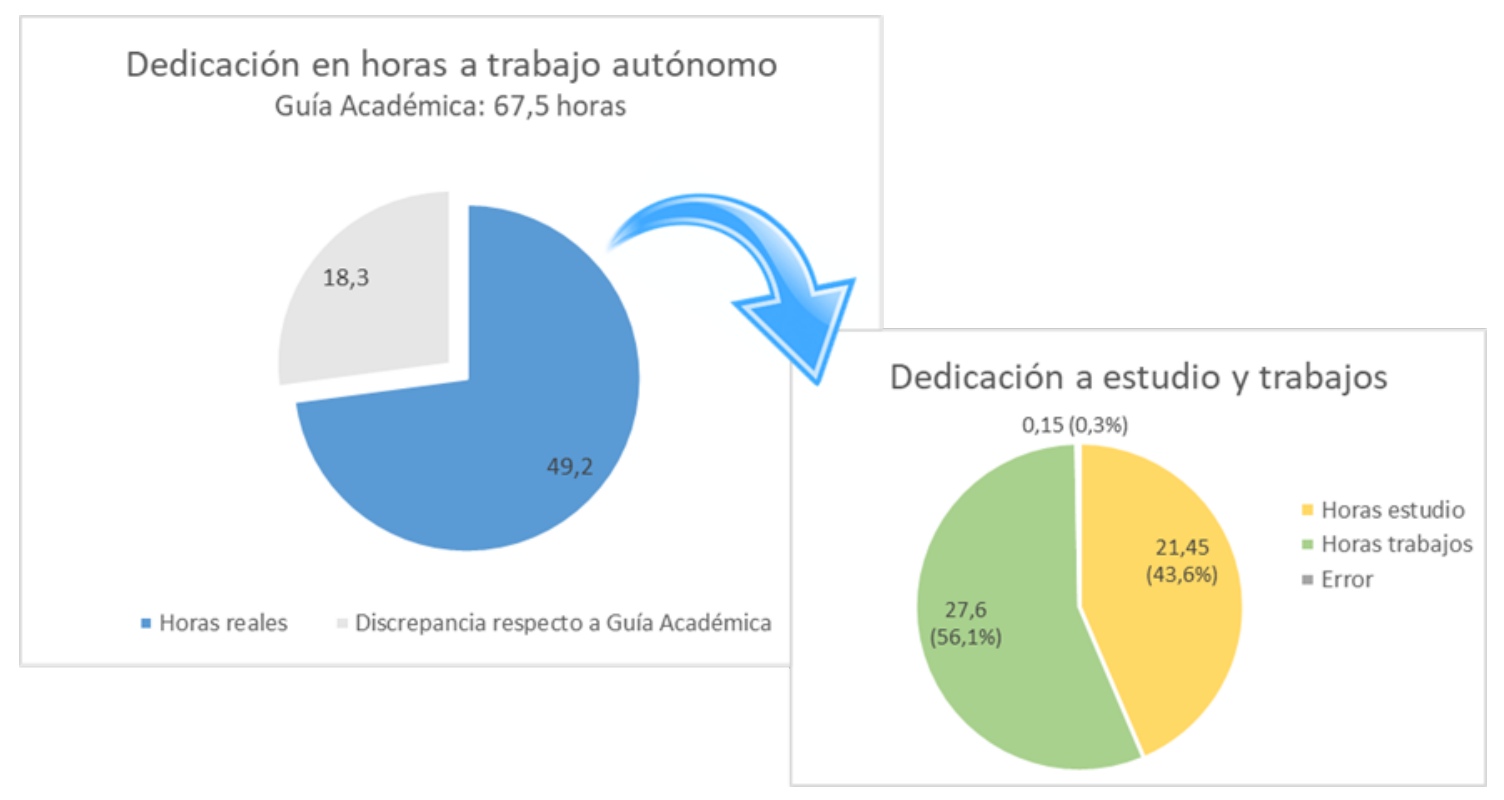

Figura 4. Volumen de trabajo autónomo real (en horas) respecto a la Guía Académica y dedicación temporal a las actividades de la asignatura.

Como indicador objetivo del impacto de esta experiencia metodológica innovadora, se compararon las calificaciones de este grupo de alumnos/as con otro grupo de 46 alumnos/as de características similares en el que no se llevó a cabo esta acción metodológica. La calificación media de los estudiantes fue de 7.7 puntos sobre 10 en ambos casos. Sin embargo, al analizar cualitativamente el desglose de puntuaciones por categorías, se observó que en el grupo en el que se empleó la metodología innovadora hubo 3 alumnos/as no presentados, 5 suspensos, 6 aprobados, 22 notables, 16 sobresalientes y 2 matrículas de honor. Sin embargo, en el grupo en el que no se implementó esta acción metodológica innovadora hubo 5 alumnos/as no presentados, 2 suspensos, 10 aprobados, 22 notables, 5 sobresalientes y 2 matrículas de honor.

\section{Discusión}

En el presente estudio se han puesto en práctica diferentes estrategias innovadoras para la adquisición de competencias en una asignatura de Psicología centrada en aspectos fisiológicos de la conducta. Esta implantación ha supuesto una percepción en el estudiantado de un nivel satisfactorio de la experiencia docente, en diferente medida en función del aspecto evaluado. La metodología docente ha sido unos de los aspectos mejor valorados, seguido de los resultados del aprendizaje y, en menor medida, de la adquisición de competencias.

Diversas razones pueden explicar, al menos en parte, la discrepancia entre las puntuaciones relativas a las competencias y a los resultados del aprendizaje. En primer lugar, los resultados del aprendizaje gozan de un mayor nivel de concreción y recuerdan a epígrafes y temáticas revisadas en los contenidos. Es posible que este hecho favorezca la percepción del alumnado de que ese resultado del aprendizaje efectivamente forma parte de la asignatura. En segundo lugar, cabe reflexionar sobre el nivel de coherencia entre ambos, competencias y resultados del aprendizaje. El concepto de competencia es múltiple y variado y, en muchos casos, dependiente del enfoque y objetivo que se persigue con la formación. En el desarrollo del Entorno Europeo de Educación Superior ha tenido una gran incidencia el proyecto Tunning Educational Structures in Europe (2003) que combina atributos que atañen al saber, saber hacer, saber estar y saber ser, además de habilidades o destrezas. Pero si el 
sentido del término es el lograr alumnos competentes en el mercado laboral, pueden utilizarse como sinónimo de nivel de capacitación, conocimientos, saberes, actitudes, capacidades y habilidades. El resultado final perseguido sería, en tal caso, alumnos y alumnas competentes "capaces no solo de acumular conocimientos, sino, sobre todo, de saber transmitir esos conocimientos y especialmente de aplicarlos con una finalidad laboral concreta" (Muñoz-Cantero y Mato-Vásquez, 2014). Pero, además, las competencias se clasifican en transversales o generales y específicas. Según RiesgoGonzález (2008), las transversales son las propias del diseño de la titulación, mientras que las específicas son el contrapunto y deben hacer explícitos los resultados de aprendizaje de una asignatura. Es posible que una reformulación de las competencias específicas de la asignatura generara un mayor nivel de coherencia entre las competencias específicas y los resultados del aprendizaje, aunque esta determinación debería implicar un estudio más exhaustivo de la asignatura y su engranaje en el conjunto de la titulación, implicando un muestreo más amplio de grupos y asignaturas.

Respecto al tiempo dedicado a la asignatura respecto al estimado para la asignatura, existe una ligera discrepancia, con una menor dedicación a la esperada. Un aspecto que podría modular estos datos es el momento en el que se ha administrado la encuesta, el penúltimo día de clase presencial, pero antes del periodo de mayor intensidad de estudio para la evaluación de la asignatura. Posiblemente se hubieran encontrado resultados diferentes a este respecto si la encuesta se hubiera administrado tras la realización del examen final de la asignatura. Cabe mencionar que, respecto a la dedicación, existe una proporción equilibrada entre el tiempo dedicado a trabajos y el dedicado al estudio.

Por último, en el desarrollo de la encuesta ha primado la brevedad, sacrificando con ello el conocimiento de la percepción del alumnado sobre cuestiones también interesantes como, por ejemplo, el nivel de conocimiento de la Guía Académica a pesar de las múltiples vías por las que se les hace llegar la información (web del Centro, disponibilidad en el Aula Virtual de la asignatura, presentación el primer día de clase, etc.). También sería conveniente considerar por qué el alumnado percibe un desajuste en el número de créditos de la asignatura. Además, sería interesante administrar la encuesta en el momento del examen para constatar posibles cambios en los datos.

Respecto a las calificaciones obtenidas por los y las estudiantes, cabe destacar que, aunque la nota media del grupo en el que se implementó la acción metodológica innovadora fue similar a la obtenida por un grupo de características semejantes en el que no se implementó dicha acción, se evidenciaron diferencias cualitativas en las calificaciones. En este sentido, en el grupo que recibió la acción metodológica innovadora, hubo mayor número de sobresalientes que en el grupo en el que no se implementó esta acción. Estos resultados son positivos y sugieren que la implementación de esta metodología innovadora de manera sistemática puede tener resultados prometedores, tanto en la satisfacción de las y los estudiantes como en el logro de resultados de aprendizaje.

Por todo ello, podemos establecer las siguientes conclusiones:

a) La implementación de metodologías docentes innovadoras en el ámbito universitario, específicamente la realización de una fase experimental en el aula, la aplicación del método del caso, el desarrollo de estrategias de ludificación y la potenciación del alineamiento docente, ha resultado satisfactoria para el estudiantado, en diferente medida en función del aspecto considerado. 
b) El aspecto mejor valorado por los alumnos y las alumnas ha sido la metodología docente, seguida del cumplimiento de los resultados de aprendizaje y, en menor medida, de la adquisición de competencias.

c) El estudiantado estima que el tiempo dedicado a la asignatura es ligeramente inferior al tiempo establecido en la Guía Académica, aunque esto podría atribuirse al momento en el que se cumplimenta la encuesta, justo antes del período de más intensidad de estudio de la asignatura.

d) Los y las estudiantes consideran que existe una proporción equilibrada entre el tiempo dedicado a trabajos y el tiempo dedicado al estudio.

e) Aunque no se han evidenciado diferencias entre el grupo que recibió la acción metodológica innovadora y otro grupo de características similares que no recibió dicha acción en la calificación media de los y las estudiantes, existen diferencias a nivel cualitativo. En esta línea, se ha encontrado una mayor proporción de sobresalientes en el grupo en el que se implementó la acción metodológica innovadora.

\section{Referencias}

Álvarez, A., González, J., Alonso, J. y Arias, J. (2014). Indicadores centinela para el plan de Bolonia. Revista de Investigación Educativa, 32(2), 327-338.

European Commission on New modes of learning and teaching in higher education (2014). Report. Luxembourg: Publications Office of the European Union.

Gil, JMS. (2001). Docencia e investigación en la universidad: una profesión, dos mundos. Educar, 28, 41-60.

Imbernon, F. (2009). Mejorar la enseñanza y el aprendizaje en la universidad. Barcelona: Ediciones Octaedro.

Kapp, K. (2012). The gamification of learning and instruction: gamebased methods and strategies for training and education. San Francisco: John Wiley \& Sons.

Muñoz- Cantero, J. y Mato-Vásquez, M.D. (2014). El proyecto docente en la universidad española según el Espacio Europeo de Educación Superior. Calidad en la educación, 40, 319-334.
Olmedo, E. (2013). Enfoques de aprendizaje de los estudiantes y metodología docente: Evolución hacia el nuevo sistema de formación e interacción propuesta en el EEES. Revista de Investigación Educativa, 31(2), 411-429.

Reynolds, Jl. (1990). El método del caso y la formación en gestión. Guía práctica. Valencia: Instituto de la Pequeña y Mediana Industria Valenciana.

Riesco-González, M. (2008). El enfoque por competencias en el EEES y sus implicaciones en la enseñanza y el aprendizaje. Tendencias Pedagógicas, 13, 79-105

Romero-Rodríguez, LM., Torres-Toukoumidis, Á. y Aguaded, I. (2017). Ludificación y educación para la ciudadanía. Revisión de las experiencias significativas. Educar, 53, 109-128.

Sancho, JM. y Hernández, F. (1997). La investigación educativa como espacio de dilemas y contradicciones. Revista de Educación, 312, 81-110.

Terenzini, PT. (1999). Research and practice in undergraduate education: And never the twain shall meet. Higher Education, 38, 33-48.

Tunning Educational Structures in Europe (2003). Informe final. Bilbao (España): Universidad de Deusto.

Zichermann, G., \& Cunningham, C. (2011). Gamification by design: Implementing game mechanics in web and mobile apps. Newton (Massachusetts): O'Reilly Media, Inc. 
Anexo

ENCUESTA ASIGNATURA PSICOENDOCRINOLOGÍA. CURSO 2017-2018. Grupo:

Esta encuesta valora diferentes aspectos de esta signatura en este grupo. Recuerda que este formulario es totalmente anónimo, responde con sinceridad según esta escala:

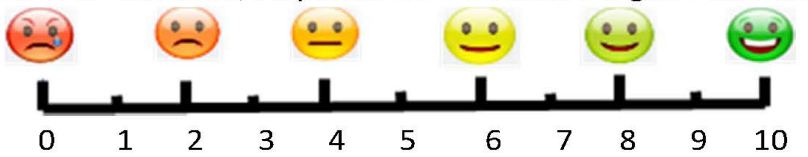

\begin{tabular}{|c|c|}
\hline Pregunta & Puntuación \\
\hline \multicolumn{2}{|c|}{ Por favor, valora en qué medida esta asignatura ha contribuido a que adquieras las siguientes competencias: } \\
\hline $\begin{array}{l}\text { Ser capaz de describir y medir variables (personalidad, inteligencia y otras aptitudes, actitudes, } \\
\text { etc.) y procesos cognitivos, emocionales, psicobiológicos y conductuales. }\end{array}$ & 01123456788910 \\
\hline Saber proporcionar retroalimentación a los destinatarios de forma adecuada y precisa & 01223456788910 \\
\hline Ser capaz de elaborar informes orales y escritos & 01123345567889910 \\
\hline Conocer y ajustarse a las obligaciones deontológicas de la Psicología & 012234556789910 \\
\hline $\begin{array}{l}\text { Conocer los procesos y etapas principales del desarrollo a lo largo del ciclo vital en sus aspectos } \\
\text { de normalidad y anormalidad }\end{array}$ & 011234556789910 \\
\hline $\begin{array}{l}\text { Conocer los fundamentos biológicos de la conducta humana y de las funciones psicológicas } \\
\text { relacionados con los procesos de pensamiento y de lenguaje }\end{array}$ & 012234567889910 \\
\hline \multicolumn{2}{|l|}{ Por favor, valora en qué medida esta asignatura ha contribuido a que aprendas lo siguiente: } \\
\hline Describir los procesos hormonales a lo largo del ciclo vital & 01125345567889910 \\
\hline $\begin{array}{l}\text { Identificar y describir los patrones hormonales asociados al dimorfismo sexual, el estrés, el } \\
\text { estado de ánimo, la conducta social y la función cognitiva. }\end{array}$ & $\begin{array}{lllllllllll}0 & 1 & 2 & 3 & 4 & 5 & 7 & 8 & 9 & 10\end{array}$ \\
\hline $\begin{array}{l}\text { Discriminar entre alteraciones psicológicas primarias y secundarias a alteraciones endocrinas } \\
\text { para establecer un diagnóstico diferencial }\end{array}$ & 01223456788910 \\
\hline Con esta asignatura, ¿̇has repetido contenidos que ya sabías de otras asignaturas? & $\square$ NO \\
\hline \multicolumn{2}{|l|}{ En caso afirmativo, ¿con qué asignaturas se han solapado contenidos? } \\
\hline \multicolumn{2}{|l|}{ En caso afirmativo, ¿qué contenidos se han repetido? } \\
\hline $\begin{array}{l}\text { ¿Cuántas horas has dedicado a la semana a trabajar y estudiar en esta asignatura, aparte de } \\
\text { las horas que estás en clase? }\end{array}$ & horas/semana \\
\hline De esas horas semanales, ¿̇uántas a estudiar? & horas/semana \\
\hline De esas horas semanales, ¿cuántas a hacer y preparar trabajos? & horas/semana \\
\hline Esta asignatura es de 4,5 ECTS, ¿̇lo consideras ajustado a la realidad? & $\square$ NO \\
\hline En caso negativo, ¿cuántos créditos ECTS crees que debería tener? & ECTS \\
\hline $\begin{array}{l}\text { ¿En qué medida crees que las metodologías docentes empleadas (preguntas de cada tema, } \\
\left.\text { Hormolimpiadas, forma de las clases, etc.) han contribuido a tu aprendizaje? }{ }^{*}\right)\end{array}$ & 01023456788910 \\
\hline ¿En qué medida crees que las prácticas de la asignatura han contribuido a tu aprendizaje? & 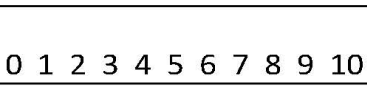 \\
\hline ¿En qué medida crees que la forma de impartir clases teóricas ha contribuido a tu aprendizaje? & 012345678910 \\
\hline \multicolumn{2}{|l|}{ ¿Qué es lo que más te ha gustado de la asignatura? } \\
\hline ¿Y & \\
\hline
\end{tabular}

$\left({ }^{*}\right)$ Ten en cuenta que las metodologías han podido variar en función del grupo al que pertenezcas, así que piensa en las que se han empleado en tu grupo. 